\title{
Distribution of integer partitions with large number of summands
}

\author{
by \\ Hsien-Kuei Hwang (Taipei)
}

1. Introduction. Let $p(n)$ denote the number of partitions of $n$ (into positive integers) and let $p(n, m)$ denote the number of partitions of $n$ into exactly $m$ parts, where the summands are counted with their multiplicities. For convenience, define $p(0)=1$ and $p(n)=0$ if $n<0$.

It is obvious that

$$
p(n, m)=p(n-m) \quad \text { for } n \geq 1 \text { and } m \geq n / 2 .
$$

We shall show that this relation holds asymptotically for a wider range of $m$ in the following sense.

Theorem 1. Let $c=\sqrt{2 / 3} \pi$ and $0<\varepsilon<1$. If $m \geq c^{-1}(1+\varepsilon) \sqrt{n} \log n$ then the relation

$$
p(n, m)=p(n-m)\left(1+O\left(n^{-\varepsilon / 2}\right)\right)
$$

holds uniformly in $m$ as $n \rightarrow \infty$.

An interesting consequence of the theorem is the following result.

Corollary 1. If $m \geq c^{-1} \sqrt{n} \log n+x \sqrt{n}$, where $x \rightarrow \infty$, then

$$
p(n, m)=p(n-m)\left(1+O\left(e^{-c x / 2}\right)\right) .
$$

The term $c^{-1} \sqrt{n} \log n$ is nothing but the asymptotic value of the mean number of summands in a random partition of $n$, each partition of $n$ being equally likely; cf. [17]. Note that the number of partitions of $n$ with $m$ 1's is given by $p(n-m)-p(n-m-1)$ for $0 \leq m \leq n$; and, consequently, $p(n-m)$ is nothing but the number of partitions of $n$ which have $\geq m 1$ 's. Thus the element 1 plays an important rôle in the counting function $p(n, m)$ when $m$ is larger than the mean value. Our aim in this paper is to show that such a phenomenon holds for more general partitions.

The proof of the theorem is rather simple and relies on a method that we previously employed in [10] (for Dirichlet series) for an analytic proof of a result of Nicolas [15]. From the formula (2), it is obvious that our method is 
based on explicitly isolating the contribution of the element 1 to the counting function $p(n, m)$. This method is quite general and can be applied to other partition problems; it can be regarded as analytic version of the sieve of Eratosthenes; cf. [14, Ch. IV]. A general theorem under a scheme due to Meinardus [13] will be derived in Section 4, which applies, in particular, to partitions into powers. Finally, we further refine the analysis to establish the limiting distribution (with convergence rate) for the number of summands in partitions into parts $\geq k, 1 \leq k=o\left(n^{1 / 4}(\log n)^{-1}\right)$, thus extending and improving the results by Auluck et al. [2].

Notation. We shall use the notation $\left[z^{n}\right] f(z)$ to represent the coefficient of $z^{n}$ in the Taylor expansion of $f(z)$. The notation $\left[u^{m} z^{n}\right] g(u, z)$ is then defined as $\left[u^{m}\right]\left(\left[z^{n}\right] g(u, z)\right)$. All limits (including $O, \ll, o$ and $\left.\sim\right)$, whenever unspecified, will be taken as $n \rightarrow \infty$. The constant $c$ always denotes $\sqrt{2 / 3} \pi$.

2. The proof of Theorem 1. Let $p^{(2)}(n, m)$ denote the number of partitions of $n$ into $m$ parts, each being $\geq 2$. Then

$$
\begin{aligned}
p(n, m) & =\sum_{0 \leq j \leq m} p^{(2)}(n-j, m-j) \\
& =\sum_{0 \leq j \leq n-m} p^{(2)}(n-m+j, j)-\sum_{1 \leq j \leq n-2 m} p^{(2)}(n+j, m+j) \\
& =p(n-m)-\sum_{1 \leq j \leq n-2 m} p^{(2)}(n+j, m+j) .
\end{aligned}
$$

Using the inequalities (cf. Lemma 1 below)

$$
p^{(2)}(n, m) \leq p(n-2 m),
$$

we obtain

$$
p(n, m)=p(n-m)+O\left(\sum_{0 \leq j<n-2 m} p(j)\right) .
$$

From the well-known asymptotic relation (cf. $[1,14])$

$$
p(n)=\frac{e^{c \sqrt{n}}}{4 \sqrt{3} n}\left(1+O\left(n^{-1 / 2}\right)\right),
$$

it follows that

$$
p(n, m)=p(n-m)\left(1+O\left(\frac{(n-m) e^{c \sqrt{n-2 m}-c \sqrt{n-m}}}{\sqrt{n-2 m}}\right)\right) .
$$

This completes the proof.

3. The generality of the method. Let $\Lambda=\left\{\lambda_{1}, \lambda_{2}, \ldots\right\}$ be a (finite or infinite) sequence of positive integers such that $1 \leq \lambda_{1}<\lambda_{2}<\ldots$ Let 
$p_{\Lambda}(n, m)=p_{\Lambda}^{(1)}(n, m)$ denote the number of partitions of $n$ into $m$ parts $\lambda_{j}$ so that

$$
\sum_{n, m} p_{\Lambda}(n, m) u^{m} z^{n}=\prod_{j \geq 1} \frac{1}{1-u z^{\lambda_{j}}} .
$$

Similarly, we define $p_{\Lambda}^{(\nu)}(n, m)$ via the generating function

$$
\sum_{n, m} p_{\Lambda}^{(\nu)}(n, m) u^{m} z^{n}=\prod_{j \geq \nu} \frac{1}{1-u z^{\lambda_{j}}} \quad(\nu=1,2, \ldots) .
$$

The inequality (3) bears the following general form.

LEMma 1. For all positive integers $n, m$ and $\mu$,

$$
p_{\Lambda}^{(\mu)}(n, m) \leq\left[z^{n-\lambda_{\mu} m}\right] \prod_{j>\mu} \frac{1}{1-z^{\lambda_{j}-\lambda_{\mu}}} .
$$

Proof. Observe that $p_{\Lambda}^{(\mu)}(n, m)$ is the number of solutions of the equations

$$
\left\{\begin{array}{l}
r_{\mu} \lambda_{\mu}+r_{\mu+1} \lambda_{\mu+1}+\ldots=n \\
r_{\mu}+r_{\mu+1}+\ldots=m
\end{array}\right.
$$

where the $r_{j}$ 's are non-negative integers; or, equivalently, the number of solutions of the equations

$$
\left\{\begin{array}{l}
r_{\mu+1}\left(\lambda_{\mu+1}-\lambda_{\mu}\right)+r_{\mu+2}\left(\lambda_{\mu+2}-\lambda_{\mu}\right)+\ldots=n-\lambda_{\mu} m \\
r_{\mu+1}+r_{\mu+2}+\ldots \leq m
\end{array}\right.
$$

The lemma follows since the number of such solutions is less than or equal to the number of partitions of $n-\lambda_{\mu} m$ into parts $\lambda_{j}-\lambda_{\mu}, j>\mu$.

Proposition 1. For any positive integer $\mu$, we have

$$
p_{\Lambda}(n, m)=\sum_{1 \leq \nu<\mu}(-1)^{\nu-1} S_{\nu}+R_{\mu}
$$

where

$$
S_{\nu}=\left[z^{n-\lambda_{\nu} m}\right]\left(\prod_{1 \leq j<\nu} \frac{z^{\lambda_{\nu}-\lambda_{j}}}{1-z^{\lambda_{\nu}-\lambda_{j}}}\right)\left(\prod_{j>\nu} \frac{1}{1-z^{\lambda_{j}-\lambda_{\nu}}}\right),
$$

and $\left|R_{\mu}\right| \leq S_{\mu}$.

Proof. (Compare [14, Thm. 4.1].) For $\mu=1$, the result reduces to Lemma 1. We suppose $\mu \geq 2$. First of all, observe that

$$
\begin{aligned}
p_{\Lambda}^{(1)}(n, m) & =\sum_{0 \leq j \leq m} p_{\Lambda}^{(2)}\left(n-\lambda_{1} j, m-j\right) \\
& =\sum_{j \geq 0} p_{\Lambda}^{(2)}\left(n-\lambda_{1} m+\lambda_{1} j, j\right)-\sum_{1 \leq j \leq \frac{n-\lambda_{2} m}{\lambda_{2}-\lambda_{1}}} p_{\Lambda}^{(2)}\left(n+\lambda_{1} j, m+j\right) .
\end{aligned}
$$


It is easily verified that for each $N \geq 0$,

$$
\sum_{j \geq 0} p_{\Lambda}^{(2)}\left(N+\lambda_{1} j, j\right)=\left[z^{N}\right] \prod_{j \geq 2} \frac{1}{1-z^{\lambda_{j}-\lambda_{1}}} .
$$

By induction we obtain (6) with

$$
\begin{aligned}
& R_{\mu}=(-1)^{\mu-1} \sum_{1 \leq j_{1} \leq \frac{n-\lambda_{2} m}{\lambda_{2}-\lambda_{1}}} \sum_{1 \leq j_{2} \leq \frac{n-\lambda_{3} m-\left(\lambda_{3}-\lambda_{1}\right) j_{1}}{\lambda_{3}-\lambda_{1}}} \cdots \\
& \sum_{1 \leq j_{\mu-1} \leq \frac{n-\lambda_{\mu} m-\left(\lambda_{\mu}-\lambda_{1}\right) j_{1}-\ldots-\left(\lambda_{\mu}-\lambda_{\mu-2}\right) j_{\mu-2}}{\lambda_{\mu}-\lambda_{\mu-1}}} p_{\Lambda}^{(\mu)}\left(n+\sum_{1 \leq i<\mu} \lambda_{i} j_{i}, m+\sum_{1 \leq i<\mu} j_{i}\right) .
\end{aligned}
$$

Applying Lemma 1, we deduce the required result.

COROllary 2. For any odd number $\nu \geq 1$ and even number $\mu \geq 2$,

$$
S_{1}-S_{2}+\ldots-S_{\mu} \leq p_{\Lambda}(n, m) \leq S_{1}-S_{2}+\ldots+S_{\nu} .
$$

Obviously, $R_{\mu}=0$ when $\mu>\mu_{0}$, where $\mu_{0}$ is the largest positive integer such that $n-\lambda_{\mu_{0}} m \geq 0$.

Corollary 3. If $m>\left(n+\lambda_{1}\right) / \lambda_{2}-1$ then

$$
p_{\Lambda}(n, m)=p_{\Lambda}^{(2)}\left(n-\lambda_{1} m\right), \quad \text { where } p_{\Lambda}^{(2)}(n)=\left[z^{n}\right] \prod_{j \geq 2} \frac{1}{1-z^{\lambda_{j}-\lambda_{1}}} .
$$

The decomposition (5) is especially useful when $m$ becomes large and $\mu=2$.

4. An analytic scheme. In this section we apply Proposition 1 to derive a result generalizing Theorem 1 using a Mellin transform technique, hereafter referred to as an analytic scheme, due to Meinardus [13]; cf. also Andrews [1, Ch. 6]. Concrete examples to which this result applies will be discussed in the next section.

Let $1 \leq \lambda_{1}<\lambda_{2}<\ldots$ be a sequence of positive integers satisfying the following conditions:

(M1) Let $D(s)=\sum_{j \geq 1} \lambda_{j}^{-s}$. The Dirichlet series $D$ converges in the halfplane $\operatorname{Re} s>\alpha>0$, and can be analytically continued into the half-plane $\operatorname{Re} s \geq-\alpha_{0}$, for some $\alpha_{0}>0$. In $\operatorname{Re} s \geq-\alpha_{0}, D$ is analytic except for a simple pole at $s=\alpha$ with residue $A$.

(M2) There exists an absolute constant $c_{1}>0$ such that $D(\sigma+i t) \ll|t|^{c_{1}}$ uniformly for $\sigma \geq-\alpha_{0}$ as $t \rightarrow \pm \infty$.

(M3) Define $g(\tau)=\sum_{j \geq 1} e^{-\lambda_{j} \tau}$, where $\tau=r+i y$ with $r>0$ and $-\pi \leq$ $y \leq \pi$. There exists a positive constant $\vartheta<1$ such that $|g(r+i y)| \leq \vartheta g(r)$ uniformly for $r \leq|y| \leq \pi$ as $r \rightarrow 0^{+}$. 
Condition (M3) is a condition of Haselgrove and Temperley [8]. Set

$$
\beta:=(A \Gamma(1+\alpha) \zeta(1+\alpha))^{1 /(\alpha+1)},
$$

where $\Gamma$ denotes the Gamma function and $\zeta$ is Riemann's zeta function, and

$$
B:=\lim _{s \rightarrow \alpha}\left(D(s)-\frac{A}{s-\alpha}\right) .
$$

Let $p_{\Lambda}^{(2)}(n)$ be defined as in Corollary 3 .

THEOREM 2. Suppose the sequence $\Lambda$ satisfies (M1)-(M3). If $\alpha=1$ $\left(\sum_{j \geq 1} \lambda_{j}^{-1}\right.$ diverges $)$, then

$$
p_{\Lambda}(n, m)=p_{\Lambda}^{(2)}\left(n-\lambda_{1} m\right)\left(1+O\left(n^{-\alpha^{\prime} / 2}+n^{-\left(\lambda_{2}-\lambda_{1}\right) \varepsilon / 2}\right)\right),
$$

uniformly for $m \geq \frac{A+\varepsilon}{2 \beta} \sqrt{n} \log n$, where $\varepsilon>0, \alpha^{\prime}=\min \left\{1, \alpha_{0}\right\}$, and

$$
p_{\Lambda}^{(2)}(n)=\xi n^{v} e^{2 \beta \sqrt{n}}\left(1+O\left(n^{-\alpha^{\prime} / 2}\right)\right),
$$

with $v=\frac{1}{4}\left(2 D(0)-5+2 A \lambda_{1}\right)$ and

$$
\xi=\frac{\lambda_{1} e^{D^{\prime}(0)+B-1}}{2 \sqrt{\pi}} \beta^{3 / 2-D(0)-A \lambda_{1}} \prod_{j \geq 2} \frac{e^{-\lambda_{1} / \lambda_{j}}}{1-\lambda_{1} / \lambda_{j}} .
$$

On the other hand, if $0<\alpha<1\left(\sum_{j \geq 1} \lambda_{j}^{-1}\right.$ converges $)$, then

(9) $p_{\Lambda}(n, m)=p_{\Lambda}^{(2)}\left(n-\lambda_{1} m\right)\left(1+O\left(n^{-\alpha^{\prime \prime} /(\alpha+1)}+e^{-\beta\left(\lambda_{2}-\lambda_{1}\right) \omega_{n}}\right)\right)$,

uniformly for $m \geq \omega_{n} n^{1 /(\alpha+1)}$, where $\omega_{n}$ is any sequence of $n$ tending to infinity, $\alpha^{\prime \prime}=\min \left\{1, \alpha, 1-\alpha, \alpha_{0}\right\}$, and

$$
p_{\Lambda}^{(2)}(n)=\eta n^{\kappa} e^{\left(1+\alpha^{-1}\right) \beta n^{\alpha /(\alpha+1)}}\left(1+O\left(n^{-\alpha^{\prime \prime} /(\alpha+1)}\right)\right),
$$

with

$$
\kappa=\frac{2 D(0)-4-\alpha}{2(\alpha+1)} \quad \text { and } \quad \eta=\frac{\lambda_{1} e^{D^{\prime}(0)}}{2 \sqrt{\pi}} \beta^{3 / 2-D(0)} \prod_{j \geq 2} \frac{1}{1-\lambda_{1} / \lambda_{j}} .
$$

Note that if $\alpha=1$, then $\beta=\sqrt{A / 6} \pi$ and $2 \beta=c \sqrt{A}$.

Pro of (sketch). We first dispose of the case when $m \geq n / \lambda_{1}$ by Corollary 3 . We next show that the two sequences

$$
\Lambda_{1}=\left\{\lambda_{j}-\lambda_{1}\right\}_{j \geq 2} \quad \text { and } \quad \Lambda_{2}=\left\{\lambda_{2}-\lambda_{1}\right\} \cup\left\{\lambda_{j}-\lambda_{2}\right\}_{j \geq 3}
$$

also satisfy (M1)-(M3). The formulae (7)-(10), apart from the error terms, follow then from Meinardus's original theorem. For techniques leading to the required (better) error terms, see Appendix.

Let $D_{1}(s)=\sum_{j \geq 2}\left(\lambda_{j}-\lambda_{1}\right)^{-s}$ and $D_{2}(s)=\left(\lambda_{2}-\lambda_{1}\right)^{-s}+\sum_{j \geq 3}\left(\lambda_{j}-\lambda_{2}\right)^{-s}$ for $\operatorname{Re} s>\alpha$. Analytic continuations of these two functions in terms of $D$ 
can be obtained in the usual way (cf. [6]). We have

$$
\begin{aligned}
D_{1}(s)= & D(s)-\lambda_{1}^{-s}+\sum_{l \geq 1}\left(\begin{array}{c}
s+l-1 \\
l
\end{array}\right) \lambda_{1}^{l}\left(D(s+l)-\lambda_{1}^{-s-l}\right), \\
D_{2}(s)= & \left(\lambda_{2}-\lambda_{1}\right)^{-s}+D(s)-\lambda_{1}^{-s} \\
& +\sum_{l \geq 1}\left(\begin{array}{c}
s+l-1 \\
l
\end{array}\right) \lambda_{2}^{l}\left(D(s+l)-\lambda_{1}^{-s-l}-\lambda_{2}^{-s-l}\right),
\end{aligned}
$$

the right-hand side providing the required analytic continuation for $\operatorname{Re} s \geq$ $-\alpha_{0}$. Note that when $0<\alpha<1$ both functions so continued have a simple pole at $s=\alpha-1$ (if $\alpha-1>-\alpha_{0}$ ); and that when $\alpha=1$ the pole at 0 is cancelled by the corresponding binomial factor $s$. Thus $\Lambda_{1}$ and $\Lambda_{2}$ satisfy (M1) with the number $\alpha_{0}$ there replaced by $\min \left\{1-\alpha, \alpha_{0}\right\}$ when $0<\alpha<1$ (and remains otherwise unaltered). These representations also guarantee that condition (M2) holds for both sequences (with a possibly different $c_{1}$ ) by the same property of $\Lambda$. Also from these representations, we deduce that

(i) if $\alpha=1$ then

$$
\begin{aligned}
& D_{1}(0)=D(0)-1+A \lambda_{1}, \quad D_{2}(0)=D(0)-1+A \lambda_{2}, \\
& D_{1}^{\prime}(0)=D^{\prime}(0)+\log \lambda_{1}+B-1-\sum_{j \geq 2}\left(\log \left(1-\frac{\lambda_{1}}{\lambda_{j}}\right)-\frac{\lambda_{1}}{\lambda_{j}}\right) ;
\end{aligned}
$$

(ii) if $0<\alpha<1$ then

$$
\begin{aligned}
& D_{1}(0)=D_{2}(0)=D(0)-1 \\
& D_{1}^{\prime}(0)=D^{\prime}(0)+\log \lambda_{1}-\sum_{j \geq 2} \log \left(1-\frac{\lambda_{1}}{\lambda_{j}}\right) .
\end{aligned}
$$

Finally, the remaining condition (M3) is easily checked. Thus, we obtain (8) and (10). Now by Proposition 1 with $\mu=2$, we have

$$
p_{\Lambda}(n, m)=p_{\Lambda}^{(2)}\left(n-\lambda_{1} m\right)+R_{2},
$$

where

$$
R_{2} \ll\left[z^{n-\lambda_{2} m}\right] \frac{1}{1-z^{\lambda_{2}-\lambda_{1}}} \prod_{j \geq 3} \frac{1}{1-z^{\lambda_{j}-\lambda_{2}}} .
$$

If $\alpha=1$ the right-hand side is bounded above, on applying Meinardus's theorem to the sequence $\Lambda_{2}$, by

$$
R_{2} \ll\left(n-\lambda_{2} m\right)^{v+A\left(\lambda_{2}-\lambda_{1}\right) / 2} e^{2 \beta \sqrt{n-\lambda_{2} m}} .
$$

From this estimate and (8), the result (7) follows. If $0<\alpha<1$ then by 
applying the same theorem

$$
R_{2} \ll\left(n-\lambda_{2} m\right)^{\kappa} \exp \left(\frac{\alpha+1}{\alpha} \beta\left(n-\lambda_{2} m\right)^{\alpha /(\alpha+1)}\right),
$$

which together with (10) implies (9).

By a similar method, we derive that the expected number of summands, where each $\Lambda$-partition of $n$ is equally likely, is asymptotic to (cf. [19])

$$
\begin{cases}\frac{\sqrt{A}}{c} \sqrt{n} \log n & \text { if } \alpha=1, \\ \frac{D(1)}{\beta} n^{1 /(\alpha+1)} & \text { if } 0<\alpha<1 .\end{cases}
$$

Furthermore, from the generating function

$$
\frac{1}{1-u z^{\lambda_{1}}} \prod_{j \geq 2} \frac{1}{1-z^{\lambda_{j}}}=\frac{1-z^{\lambda_{j}}}{1-u z^{\lambda_{1}}} \prod_{j \geq 1} \frac{1}{1-z^{\lambda_{j}}},
$$

it follows that the number of $\Lambda$-partitions of $n$ in which there are $\geq m \lambda_{1}$ 's is given by $p_{\Lambda}\left(n-\lambda_{1} m\right)$, where $p_{\Lambda}(n)$ is the total number of $\Lambda$-partitions of $n$ (cf. $[13,1])$ :

$$
\begin{aligned}
p_{\Lambda}(n) & =\left[z^{n}\right] \prod_{j \geq 1} \frac{1}{1-z^{\lambda_{j}}} \\
& =\varrho n^{\sigma} e^{(1+1 / \alpha) \beta n^{\alpha /(\alpha+1)}}\left(1+O\left(n^{-\min \left\{1, \alpha, \alpha_{0}\right\} /(\alpha+1)}\right)\right),
\end{aligned}
$$

with

$$
\sigma=\frac{2 D(0)-2+\alpha}{2(\alpha+1)} \text { and } \varrho=\frac{e^{D^{\prime}(0)} \beta^{1 / 2-D(0)}}{\sqrt{2 \pi(\alpha+1)}} .
$$

Since the error term in (11) is better than that in Meinardus's original paper, a sketch of the proof is given in the Appendix.

5. Applications. Consider first the case when $\lambda_{j}=j+k-1$, where $k \geq 1$ is a fixed integer. The assumptions of Theorem 2 being easily checked with $\alpha=1$, we obtain $\left(^{1}\right)$

$$
p_{\Lambda}(n, m)=p(n-k m)\left(1+O\left(n^{-\varepsilon / 2}\right)\right),
$$

uniformly for $m \geq c^{-1}(1+\varepsilon) \sqrt{n} \log n, 0<\varepsilon<1$, and for each fixed $k$. Thus removing from the set of positive integers the first $k-1$ numbers does not change drastically the asymptotic behaviour of $p_{\Lambda}$ in the specified range.

$\left({ }^{1}\right)$ The quantities $p_{\Lambda}$, when written without their subscript, have $\mathbb{Z}^{+}$as their underlying set. 
Note that in this case a direct application of Proposition 1 using (4) is simpler. Moreover, the condition that $k=O(1)$ can be relaxed and we can show that

$$
p_{\Lambda}(n, m)=p(n-k m)\left(1+O\left(n^{-\varepsilon / 2} e^{-k(\log n)^{2} /(4 c \sqrt{n})}\right)\right),
$$

for $m \geq c^{-1}(1+\varepsilon) \sqrt{n} \log n$ and $1 \leq k=o\left(\varepsilon \sqrt{n}(\log n)^{-1}\right)$.

For examples for which $A \neq 1$, take $D(s)=\left(1-k^{-s}\right) \zeta(s)$, where $k \geq 3$. Then our theorem applies with $A=(k-1) / k$.

As another application of Theorem 2, consider the case when $\lambda_{j}=j^{k}$, where $k$ is a fixed positive integer $\geq 2$. The conditions (M1)-(M3) being satisfied as in [8] (using estimates from Waring's problem), we obtain

$$
p_{\Lambda}(n, m)=p_{\Lambda}^{(2)}(n-m)\left(1+O\left(n^{-1 /(k+1)}+e^{-\beta\left(2^{k}-1\right) \omega_{n}}\right)\right),
$$

uniformly for

$$
m \geq \omega_{n} n^{k /(k+1)} \quad \text { with } \quad \beta=\left(\frac{1}{k} \Gamma(1+1 / k) \zeta(1+1 / k)\right)^{k /(k+1)},
$$

where $\omega_{n} \rightarrow \infty$ and

$$
p_{\Lambda}^{(2)}(n)=\gamma_{k} n^{-(5 k+1) /(2 k+2)} e^{(1+k) \beta n^{1 /(k+1)}}\left(1+O\left(n^{-1 /(k+1)}\right)\right),
$$

with

$$
\gamma_{k}=(2 \pi)^{-(k+1) / 2}(1+1 / k)^{-1 / 2} \beta^{2} \prod_{j \geq 2} \frac{1}{1-j^{-k}} .
$$

Note that the expected number of summands is asymptotic to (cf. [19])

$$
\frac{\zeta(k)}{\beta} n^{k /(k+1)}\left(1+O\left(n^{-1 /(k+1)}\right)\right),
$$

for $k \geq 2$.

6. Cases of failure. In this section we show by two examples that although conditions (M1)-(M3) are rather restrictive, results similar to Theorem 2 may hold for more general partitions. From the above discussions, we naturally predict for general $\Lambda$-partitions that

$$
p_{\Lambda}(n, m) \sim\left[z^{n-\lambda_{1} m}\right] \prod_{j \geq 2} \frac{1}{1-z^{\lambda_{j}-\lambda_{1}}},
$$

for $m$ in a certain range (lying to the right of the mean value), even without explicit representation of the asymptotic behaviour of the right-hand side in terms of elementary functions as in the results of the last section. This is the case for, say, partitions into primes.

Take first $\lambda_{j}=k(j-1)+h$, where $k \geq 2,1 \leq h<k$ and $(h, k)=1$. Then $p_{\Lambda}(n, m)=0$ whenever $n-h m \not \equiv 0(\bmod k)$. Applying $(5)$, we obtain 
by the derivations of $(2)$

$$
p_{\Lambda}(n, m)=p((n-h m) / k)\left(1+O\left(n^{-\varepsilon / 2}\right)\right),
$$

for $m \geq \frac{1+\varepsilon}{\sqrt{k} c} \sqrt{n} \log n$ and for $n-h m \equiv 0(\bmod k)$, the $O$-term depending on $k$. We can of course let $k \rightarrow \infty$ with $n$ and the relation still holds provided that $k=o(\sqrt{n})$.

Next consider the Mahler partition $\lambda_{j}=2^{j-1}$. Let

$$
p_{\Lambda}^{(2)}(n)=\left[z^{n}\right] \prod_{j \geq 1} \frac{1}{1-z^{2^{j}-1}} .
$$

Then by (5), we have $p_{\Lambda}(n, m)=p_{\Lambda}^{(2)}(n-m)+R_{2}$ where

$$
\left|R_{2}\right| \leq\left[z^{n-2 m}\right] \frac{z}{1-z} \prod_{j \geq 2} \frac{1}{1-z^{2^{j}-2}}=\sum_{0 \leq j \leq(n-1) / 2-m} p_{\Lambda}^{(2)}(j) .
$$

Using standard analytic methods as in $[3,18]$ or the Tauberian theorems in $[16,21]$, we can verify that $p_{\Lambda}(n, m) \sim p_{\Lambda}^{(2)}(n-m)$ whenever $(m \log n) / n \rightarrow$ $\infty$. Note that the expected number of summands in a random Mahler partition is asymptotic to (cf. [18]) $(2 \log 2) n / \log n$, each partition of $n$ being assigned the same probability.

7. Limiting distributions. In this section we show that the above method is also suitable for deriving limiting distribution of the number of summands. For simplicity we consider only the case when $\lambda_{j}=j+k-1$, that is, $\Lambda$ is the set of positive integers $\geq k$. For further results on the limiting distribution of the number of summands in partitions, see $[8,22-25,12$, $20,7]$.

Let $\xi_{n}^{(k)}$ represent the number of summands (counted with multiplicities) in a random $\Lambda$-partition of $n$ (i.e., into parts $\geq k$ ), each $\Lambda$-partition of $n$ being equally likely. Auluck et al. [2] showed, by a method due to Erdős and Lehner [5], that

$$
\operatorname{Pr}\left\{\xi_{n}^{(1)}=m\right\}=\frac{p_{\Lambda}(n, m)}{p(n)} \sim n^{-1 / 2} \exp \left(-\frac{c}{2} x-\frac{2}{c} \exp \left(-\frac{c}{2} x\right)\right),
$$

uniformly for $m=c^{-1} \sqrt{n} \log n+x \sqrt{n}, x=O(1)$. The distribution on the right-hand side is an extreme-value distribution; cf. [25]. We shall further refine the analysis in Section 2 to obtain the following result which is more general and precise than (12).

Theorem 3. If $m=c^{-1} \sqrt{n} \log n+x \sqrt{n}$ then

$$
\begin{aligned}
& \operatorname{Pr}\left\{\xi_{n}^{(k)}=m\right\} \\
= & \frac{(2 / c)^{k-1}}{(k-1) ! \sqrt{n}} \exp \left(-\frac{c}{2} k x-\frac{2}{c} \exp \left(-\frac{c}{2} x\right)\right)\left(1+O\left(\frac{k^{2}(\log n)^{2}}{\sqrt{n}}\right)\right),
\end{aligned}
$$


uniformly for all $x$ and for $1 \leq k=o\left(n^{1 / 4}(\log n)^{-1}\right)$. Furthermore, if $k \rightarrow$ $\infty$, where $k=o\left(n^{1 / 4}(\log n)^{-1}\right)$, then for

$$
m=\frac{2 \sqrt{n}}{c} \log \frac{2 \sqrt{n}}{c k}+x \frac{2 \sqrt{n}}{c \sqrt{k}} \quad(x=O(1)),
$$

we have

$$
\operatorname{Pr}\left\{\xi_{n}^{(k)}=m\right\}=\frac{e^{-x^{2} / 2}}{\sqrt{2 \pi} \sigma_{n, k}}\left(1+O\left(\frac{x^{3}}{\sqrt{k}}+\frac{k^{2}(\log n)^{2}}{\sqrt{n}}\right)\right),
$$

uniformly in $k$, where $\sigma_{n, k}=2 \sqrt{n} /(c \sqrt{k})$.

Note that when $k=1$, the result (13) may be derived from the general formulae of Szekeres [24, 25]; cf. also [4]. Once we have started with (5), the remaining derivations become more or less standard; thus details will be omitted.

Pro of (sketch). By Proposition 1, we have for any $\mu \geq 1$,

$$
p(n, m)=\sum_{1 \leq \nu \leq \mu}(-1)^{\nu-1}\left[z^{n-(\nu+k-1) m}\right] f_{\nu}(z)+R_{\mu+1},
$$

where

$$
f_{\nu}(z)=\left(\prod_{1 \leq k<\nu} \frac{z^{k}}{1-z^{k}}\right)\left(\prod_{k \geq 1} \frac{1}{1-z^{k}}\right)
$$

and $\left|R_{\mu+1}\right| \leq\left[z^{n-(\mu+k) m}\right] f_{\mu+1}(z)$. Set

$$
N=\pi^{-1} \sqrt{6(n-(\nu+k-1) m)}
$$

and write $z=e^{-\tau}$, where $\tau=N^{-1}+i \theta,|\theta| \leq \pi$. It can be verified that (cf. $[1, \mathrm{Ch} . \mathrm{VI}])$

$$
f_{\nu}\left(e^{-\tau}\right)=\frac{\tau^{-\nu+3 / 2} e^{\pi^{2} /(6 \tau)}}{\sqrt{2 \pi}(\nu-1) !}\left(1+O\left(\nu^{2}|\tau|\right)\right),
$$

uniformly for $1 \leq \nu=o\left(n^{1 / 4}\right)$ and $|\theta| \leq N^{-1}$. On the other hand, we have

$$
\left|f_{\nu}\left(e^{-\tau}\right)\right|<f_{\nu}\left(e^{-1 / N}\right) e^{-N / 20},
$$

for $N^{-1}<|\theta| \leq \pi$. This can be seen as follows. Obviously,

$$
\left|\log f_{\nu}(z)\right| \leq \log f_{\nu}(|z|)-\frac{|z|}{1-|z|}\left(1-\frac{1-|z|}{|1-z|}\right) .
$$

Now if $N^{-1}<|\theta| \leq \pi$ then

$$
\frac{1-|z|}{|1-z|}=\frac{1-e^{-1 / N}}{\sqrt{\left(1-e^{-1 / N}\right)^{2}+2 e^{-1 / N}(1-\cos \theta)}} \leq\left(1+\frac{4 e}{\pi^{2}(e-1)^{2}}\right)^{-1 / 2},
$$

where we have used the inequalities $1-\cos \theta \geq 2 \theta^{2} / \pi^{2}$ for $|\theta| \leq \pi$, and 
$x^{2} e^{-x}\left(1-e^{-x}\right)^{-2} \geq e(e-1)^{-2}$ for $0 \leq x \leq 1$. Now using the inequality $e^{-1 / N}\left(1-e^{-1 / N}\right)^{-1} \geq N / e$ for $N \geq 1$, we obtain

$$
\frac{|z|}{1-|z|}\left(1-\frac{1-|z|}{|1-z|}\right) \geq \frac{N}{e}\left(1-\left(1+\frac{4 e}{\pi^{2}(e-1)^{2}}\right)^{-1 / 2}\right)>\frac{N}{20},
$$

as required. From the two estimates (17) and (18), we deduce, by Laplace's method, that

$$
\begin{aligned}
{\left[z^{n-(\nu+k-1) m}\right] f_{\nu}(z)=} & \frac{(2 / c)^{\nu-1}(n-(\nu+k-1) m)^{(\nu-3) / 2}}{4 \sqrt{3}(\nu-1) !} e^{c \sqrt{n-(\nu+k-1) m}} \\
& \times\left(1+O\left(\frac{\nu^{2}}{\sqrt{n-(\nu+k-1) m}}\right)\right),
\end{aligned}
$$

for $1 \leq \nu=o\left(n^{1 / 4}\right)$. In view of the asymptotic formula (cf. also [4])

$$
\begin{aligned}
p_{\Lambda}(n) & =\left[z^{n}\right] \prod_{j \geq k} \frac{1}{1-z^{j}} \\
& =\frac{(k-1) !}{4 \sqrt{3}}\left(\frac{c}{2}\right)^{k-1} n^{-(k+1) / 2} e^{c \sqrt{n}}\left(1+O\left(k^{2} n^{-1 / 2}\right)\right) \quad\left(k=o\left(n^{1 / 4}\right)\right),
\end{aligned}
$$

obtained by applying, say, the saddle-point method, we have

$$
\begin{aligned}
p_{\Lambda}(n)^{-1}\left[z^{n-(\nu+k-1) m}\right] f_{\nu}(z) & \\
= & \frac{(2 / c)^{\nu+k-2}}{(k-1) !(\nu-1) !} n^{(\nu+k-2) / 2} e^{-c(\nu+k-1) m /(2 \sqrt{n})} \\
& \times\left(1+O\left(\frac{k^{2}+\nu^{2}}{\sqrt{n}}+\frac{\nu(\nu+k) m}{n}+\frac{(\nu+k)^{2} m^{2}}{n^{3 / 2}}\right)\right) .
\end{aligned}
$$

Thus for $m=c^{-1} \sqrt{n} \log n+x \sqrt{n}$ and $k=o\left(n^{1 / 4}(\log n)^{-1}\right)$, we obtain

$$
\begin{array}{r}
p_{\Lambda}(n)^{-1}\left[z^{n-(\nu+k-1) m}\right] f_{\nu}(z) \\
=\frac{(2 / c)^{\nu+k-2} e^{-c(\nu+k-1) x / 2}}{(k-1) !(\nu-1) ! \sqrt{n}}\left(1+O\left(\frac{\nu^{2}+k^{2}}{\sqrt{n}}(\log n)^{2}\right)\right) .
\end{array}
$$

From this relation and (16), (13) follows (by choosing a sufficiently large $\mu$ in (16)). Similarly, substituting (14) into (19), we deduce (15).

The theorem is comparable with the results of Fristedt [7] for the limiting distribution of the size of the $k$ th largest summand ( $Y_{k}$, in his notation) in a random partition of $n$ (integers $\geq 1$ ). Actually, the two quantities have, by conjugacy, the same distribution. Although his range of validity of (15) for $k$ is slightly larger than ours $\left(k=o\left(n^{1 / 4}\right)\right)$, our local limit theorems (13) and (15) are stronger than his global limit theorems (without convergence rates). 
We can rewrite the formula (13) in a different form as follows. If

$$
m=\frac{2 \sqrt{n}}{c} \log \frac{2 \sqrt{n}}{c y}, \quad y>0,
$$

then

$$
\operatorname{Pr}\left\{\xi_{n}^{(k)}=m\right\}=\frac{c k}{2 \sqrt{n}} \cdot \frac{y^{k} e^{-y}}{k !}\left(1+O\left(\frac{k^{2}(\log n)^{2}}{\sqrt{n}}\right)\right),
$$

a gamma distribution.

8. Concluding remarks. One may extend Theorem 3 to general partitions under Meinardus's (or other) scheme, the result obtained being comparable to those in $[8,12,20]$. One may also consider the case of repeated $\lambda_{j}$, that is, the sequence $\Lambda$ satisfies $1 \leq \lambda_{1} \leq \lambda_{2} \leq \ldots$, the computations involved being more complicated.

The method used here is also suitable for the quantities studied by Szalay and Turán in $[22,23]$. The generating functions there are of the forms

$$
\left(\prod_{1 \leq j<k} \frac{1}{1-z^{j}}\right)\left(\prod_{j \geq k} \frac{1}{1-u z^{j}}\right) \text { and }\left(\prod_{1 \leq j \leq k} \frac{1}{1-u z^{j}}\right)\left(\prod_{j>k} \frac{1}{1-z^{j}}\right) \text {, }
$$

where $k \geq 1$.

It should be mentioned, as emphasized in [9], that if the multiplicity of each summand is counted only once, or if each part is allowed to appear at most once, then the limiting distributions of the number of summands are Gaussian for a large class of partitions. For more quantitative results, see $[9]$.

General combinatorial inequalities for $p_{\Lambda}(n, m)$ are developed in [11], which are especially useful for partitions with a small number of summands.

Appendix. In this appendix, we sketch the proof of (11); details can be found in [9]. In Meinardus's original paper, the error term for $p_{\Lambda}(n)$ in (11) is of the form $O\left(n^{-\kappa_{1}}\right)$, where (cf. $[13,1]$ )

$$
\kappa_{1}=\frac{\alpha}{\alpha+1} \min \left\{\frac{\min \left\{1, \alpha_{0}\right\}}{\alpha}-\frac{\delta}{4}, \frac{1}{2}-\delta\right\},
$$

$\delta$ being an arbitrary positive number.

Set

$$
F(z)=\prod_{j \geq 1}\left(1-z^{\lambda_{j}}\right)^{-1} .
$$

Write $z=e^{-\tau}=e^{-r-i y}$, where $r>0$ and $-\pi \leq y \leq \pi$. By Mellin transform (cf. $[13,1]$ ), we have

$$
F\left(e^{-\tau}\right)=e^{D^{\prime}(0)} \tau^{-D(0)} e^{A \Gamma(\alpha) \zeta(\alpha+1) \tau^{-\alpha}}\left(1+O\left(\left|\tau^{\alpha^{\prime}}\right|\right)\right),
$$


uniformly for $|y| \leq r$, as $r \rightarrow 0^{+}$, where $\alpha^{\prime}=\min \left\{1, \alpha_{0}\right\}$. On the other hand, by our assumption (M3), we deduce that

$$
\frac{\left|F\left(e^{-r-i y}\right)\right|}{F\left(e^{-r}\right)} \ll e^{-c_{2} r^{-\alpha}} \quad\left(c_{2}>0\right),
$$

uniformly for $r \leq|y| \leq \pi$. From the estimate (20), it follows that

$$
\frac{\left|F\left(e^{-r-i y}\right)\right|}{F\left(e^{-r}\right)} \ll e^{-c_{3} r^{-\alpha / 7}} \quad\left(c_{3}>0\right),
$$

uniformly for $r^{1+3 \alpha / 7} \leq|y| \leq r$, as $r \rightarrow 0^{+}$. Choose now

$$
r=\left(\frac{A \alpha \Gamma(\alpha) \zeta(\alpha+1)}{n}\right)^{1 /(\alpha+1)} .
$$

By Cauchy's formula using (20)-(23), we have

$$
p_{\Lambda}(n)=I_{1}+I_{2}+O\left(F\left(e^{-r}\right) e^{n r-c_{4} n^{\alpha /(7 \alpha+7)}}\right),
$$

where

$$
I_{1}=\frac{e^{D^{\prime}(0)+n r}}{2 \pi} r^{1-D(0)} \int_{|t| \leq r^{3 \alpha / 7}}(1+i t)^{-D(0)} e^{i n r t+A \Gamma(\alpha) \zeta(\alpha+1) r^{-\alpha}(1+i t)^{-\alpha}} d t,
$$

and

(25) $I_{2} \ll r^{1-D(0)+\alpha^{\prime}} e^{n r} \int_{|t| \leq r^{3 \alpha / 7}}|1+i t|^{-D(0)} e^{A \Gamma(\alpha) \zeta(\alpha+1) r^{-\alpha} \operatorname{Re}(1+i t)^{-\alpha}} d t$

$$
\ll r^{1-D(0)+\alpha^{\prime}+\alpha / 2} e^{n r+A \Gamma(\alpha) \zeta(\alpha+1) r^{-\alpha}},
$$

where we used the inequalities

$$
\operatorname{Re}(1+i t)^{-\alpha} \leq\left(1+t^{2}\right)^{-\alpha / 2} \leq 1-\left(1-2^{-\alpha / 2}\right) t^{2} \quad \text { for }-1 \leq t \leq 1 .
$$

For $I_{1}$, setting $b=\sqrt{\alpha(\alpha+1) A \Gamma(\alpha) \zeta(\alpha+1)}$ and making the change of variables $v^{2}=b^{2} r^{-\alpha} t^{2}$, we obtain

$$
I_{1}=\frac{e^{D^{\prime}(0)+n r+A \Gamma(\alpha) \zeta(\alpha+1) r^{-\alpha}}}{2 \pi b} r^{1-D(0)+\alpha / 2} \int_{-b r^{-\alpha / 7}}^{b r^{-\alpha / 7}} e^{-v^{2} / 2} T_{r}(v) d v,
$$

where

$$
T_{r}(v)=1+\frac{(\alpha+2) v^{2}-6 D(0)}{6 b} r^{\alpha / 2} i v+O\left(\left(v^{6}+v^{2}\right) r^{\alpha}\right)
$$

from which we deduce that

$$
I_{1}=\frac{e^{D^{\prime}(0)+n r+A \Gamma(\alpha) \zeta(\alpha+1) r^{-\alpha}}}{\sqrt{2 \pi} b} r^{1-D(0)+\alpha / 2}\left(1+O\left(r^{\alpha}\right)\right) .
$$

Thus (11) follows from (23)-(26). 


\section{References}

[1] G. E. Andrews, The Theory of Partitions, Encyclopedia Math. Appl. 2, AddisonWesley, 1976.

[2] F. C. Auluck, S. Chowla and H. Gupta, On the maximum value of the number of partitions of $n$ into $k$ parts, J. Indian Math. Soc. 6 (1942), 105-112.

[3] N. G. de Bruijn, On Mahler's partition problem, Indag. Math. 10 (1948), 210-220.

[4] J. Dixmier et J.-L. Nicolas, Partitions sans petits sommants, in: A Tribute to Paul Erdős, A. Baker, B. Bollobás and A. Hajnal (eds.), Cambridge University Press, 1990, 120-152.

[5] P. Erdös and J. Lehner, The distribution of the number of summands in the partitions of a positive integer, Duke Math. J. 8 (1941), 335-345.

[6] P. Flajolet, X. Gourdon and P. Dumas, Mellin transforms and asymptotics: harmonic sums, Theoret. Comput. Sci. 144 (1995), 3-58.

[7] B. Fristedt, The structure of random partitions of large integers, Trans. Amer. Math. Soc. 337 (1993), 703-735.

[8] C. B. Haselgrove and H. N. V. Temperley, Asymptotic formulae in the theory of partitions, Proc. Cambridge Philos. Soc. 50 (1954), 225-241.

[9] H.-K. Hwang, Limit theorems for the number of summands in integer partitions, submitted.

[10] —, Sur la répartition des valeurs des fonctions arithmétiques, I. Le nombre de facteurs premiers d'un entier, submitted.

[11] H.-K. Hw ang and Y.-N. Y eh, Distribution of integer partitions with a small number of summands, in preparation.

[12] D. V. Lee, The asymptotic distribution of the number of summands in unrestricted A-partitions, Acta Arith. 65 (1993), 29-43.

[13] G. Meinardus, Asymptotische Aussagen über Partitionen, Math. Z. 59 (1954), 388-398.

[14] W. Narkiewicz, Number Theory, World Scientific, Singapore, 1983 (translated by S. Kanemitsu).

[15] J.-L. Nicolas, Sur la distribution des nombres ayant une quantité fixée de facteurs premiers, Acta Arith. 44 (1984), 191-200.

[16] W. B. Pennington, On Mahler's partition problem, Ann. of Math. 57 (1953), 531-546.

[17] L. B. Richmond, The moments of partitions, I, Acta Arith. 26 (1975), 411-425.

[18] —, Mahler's partition problem, Ars Combin. 2 (1976), 169-189.

[19] -, The moments of partitions, II, Acta Arith. 28 (1975), 229-243.

[20] - Some general problems on the number of parts in partitions, ibid. 66 (1994), 297-313.

[21] W. Schwarz, Einige Anwendungen Tauberscher Sätze in der Zahlentheorie. C. Mahler's Partitionsproblem, J. Reine Angew. Math. 228 (1967), 182-188.

[22] M. Szalay and P. Turán, On some problems of the statistical theory of partitions with application to characters of the symmetric group I, Acta Math. Acad. Sci. Hungar. 29 (1977), 361-379.

[23] - - - On some problems of the statistical theory of partitions with application to characters of the symmetric group II, ibid., 381-392. 
[24] G. Szekeres, Some asymptotic formulae in the theory of partitions II, Quart. J. Math. Oxford 4 (1953), 96-111.

[25] - Asymptotic distribution of partitions by number and size of parts, in: Number Theory, Colloq. Math. Soc. János Bolyai 51, North-Holland, 1987, 527-538.

Institute of Statistical Science

Academia Sinica

Taipei 115, Taiwan

E-mail: hkhwang@stat.sinica.edu.tw

Received on 27.3.1996

and in revised form on 16.8.1996 